\title{
MARVEL: MEASURED ACTIVE ROTATIONAL-VIBRATIONAL ENERGY LEVELS
}

\author{
Tibor Furtenbacher, ${ }^{a}$ Attila G. Császár, ${ }^{\mathrm{a}, *}$ and Jonathan Tennyson ${ }^{\mathrm{b}}$ \\ ${ }^{a}$ Laboratory of Molecular Spectroscopy, Institute of Chemistry, \\ Eötvös University, P.O. Box 32, H-1518 Budapest 112, Hungary \\ ${ }^{\mathrm{b}}$ Department of Physics and Astronomy, University College London, \\ Gower Street, London WC1E 6BT, United Kingdom
}

\begin{abstract}
An algorithm is proposed, based principally on an earlier proposition of Flaud and coworkers [Mol. Phys. 32 (1976) 499], that inverts the information contained in uniquely assigned experimental rotational-vibrational transitions in order to obtain measured active rotational-vibrational energy levels (MARVEL). The procedure starts with collecting, critically evaluating, selecting, and compiling all available measured transitions, including assignments and uncertainties, into a single database. Then, spectroscopic networks (SN) are determined which contain all interconnecting rotational-vibrational energy levels supported by the grand database of the selected transitions. Adjustment of the uncertainties of the lines is performed next, with the help of a robust weighting strategy, until a self-consistent set of lines and uncertainties is achieved. Inversion of the transitions through a weighted leastsquares-type procedure results in MARVEL energy levels and associated uncertainties. Local sensitivity coefficients could be computed for each energy level. The resulting set of MARVEL levels is called active as when new experimental measurements become available the same evaluation, adjustment, and inversion procedure should be repeated in order to obtain more dependable energy levels and uncertainties. MARVEL is tested on the example of the $\mathrm{H}_{2}{ }^{17} \mathrm{O}$ isotopologue of water and a list of 2736 dependable energy levels, based on 8369 transitions, has been obtained.
\end{abstract}

Keywords: MARVEL, rotational-vibrational energy levels, robust fitting, $\mathrm{H}_{2}{ }^{17} \mathrm{O}$, uncertainties. 


\section{Introduction}

There are several areas in the sciences where experimentally measured quantities, with well-defined uncertainties, and quantities preferred on some theoretical ground, again with appropriate uncertainties, are decidedly distinct but relations can be worked out between the two sets of data. One such area is thermochemistry [1,2]. Here the preferred quantities required by modelers using thermochemical data, and thus entered into appropriate databases, are the enthalpies of formation of the species in question. On the other hand, the measured quantities from which the enthalpies of formation have been derived comprise many quantities of drastically different origin, including spectroscopic measurements of dissociation energies, appearance energies, energy quantities in positive ion cycles, etc. [2]. Another area of high interest is reaction kinetics [3,4]. In reaction kinetics it is usual to compare outcomes of relevant experiments with outcomes of sometimes complicated reaction models with fixed and variable reaction rate constants and perhaps other parameters. Both the models and the model parameters are chosen according to the best available knowledge but if the modeling results differ from the experimental ones it is often impossible to decide whether the model chosen is incomplete, the model parameters are inaccurate, or the interpretation (or even the execution) of the experiment is incorrect. The rate constants are needed by modelers to check the suitability of their models and rate constants of the elementary reactions should be transferable within different reaction mechanisms. In the third field of most interest for this study, in molecular spectroscopy, transitions are measured but it is preferable to deduce energy levels from the measurements $[5,6,7]$, for example, when partition functions are to be determined via direct summation.

In all the cases mentioned a certain inversion of the experimentally measured quantities is needed. Since in spectroscopy the relation between the measurable transitions and the theoretically available energy levels is linear and exceedingly simple, it is not surprising that it was in spectroscopy where, to the best of our knowledge, proposals for this inversion process have been advanced, perhaps first by Flaud and co-workers [5]. In reaction kinetics Frenklach and co-workers $[3,4]$ proposed a parameter optimization scheme which, however, has not found widespread acceptance and use. The basic reason behind this is that the relation between the model parameters and the model outcomes is decidedly nonlinear. In thermochemistry, Ruscic and co-workers proposed the Active Thermochemical Tables (ATcT) approach [1] for obtaining accurate enthalpies of formation with improved 
uncertainties by using all available information in a well-defined thermochemical network. Here again the great advantage is that the appropriate inversion relations are linear.

The principal purpose of the present paper is to advance the procedure proposed by Flaud and co-workers [5] and build a set of measured active rotational-vibrational energy levels using all the available spectroscopic information in one grand inversion and refinement process. The rotational-vibrational energy levels are considered measured as they are obtained from experiment as opposed to their determination through high-accuracy quantum chemical computations (see, e.g., Ref. 8). The set of measured energy levels is called active in the sense of the ATcT approach [1], and implies that if new experimental transitions become available the refinement must be carried out over and over again and a new set of rotational-vibrational energy levels with improved estimates for positions and uncertainties should be obtained. The name we coined for the process is therefore MARVEL, standing for Measured Active Rotational-Vibrational Energy Levels.

We have built MARVEL on Ref. [5], the ATcT [1] approach, and the ideas of Frenklach and co-workers [3,4] and Watson [9]. Clearly, the underlying least-squares refinement procedures can be applied quite generally. Nevertheless, in what follows the emphasis is on the spectroscopic application so methodological details are given only for this special case. To test the procedure and the code developed, results are reported for the water isotopologue $\mathrm{H}_{2}{ }^{17} \mathrm{O}$, as a first relatively simple application of MARVEL. $\mathrm{H}_{2}{ }^{17} \mathrm{O}$ was chosen as it contains a relatively small number of accurately measured transitions (on the order of 8000 ), including a large number of rotational transitions on the ground vibrational state, and water is probably the single most important polyatomic molecule whose spectroscopy on the ground electronic state is especially relevant in a number of applications, including understanding of the greenhouse effect, astrophysical environments, etc.

\section{Methodological Details}

In this Section we present a short summary of the simple weighted least-squares theory behind MARVEL. The discussion is basically built upon that of Flaud and co-workers [5]. The small but significant differences between their approach and that applied here are emphasized in greater detail.

Determination of the measured energy levels is based principally on the following steps: 
(1) Collection, validation (critical evaluation and selection), and compilation of all available measured transitions, including their systematic and unique assignments and uncertainties, into a single database.

(2) Based on the given database of assigned transitions, determination of those energy levels of the given species which belong to a particular spectroscopic network (SN).

(3) Cleansing of the database, i.e., deletion of all clearly incorrect (e.g., misassigned, misidentified, or mislabeled) transitions from the database.

(4) Within a given SN, setting up of a vector containing all the experimentally measured transitions selected, another one comprising the requested MARVEL energy levels, and a matrix which describes the relation between the transitions and the energy levels.

(5) Solution of the resulting set of linear equations corresponding to the chosen pair of vectors and the inversion (design) matrix. During solution of the set of linear equations uncertainties in the measured transitions can be incorporated which result in uncertainties of the energy levels determined.

If the grand database of transitions contains more than one $\mathrm{SN}$, the absolute energy levels of the higher SN(s) can only be obtained if the value of the lowest energy level within the SN, with zero uncertainty, is set up correctly.

\subsection{Dataset}

The principal input to MARVEL is a grand list of $N_{\mathrm{t}}$ experimentally measured transitions. The required data include wavenumbers, $\sigma_{i}$, together with their associated uncertainties, $\delta_{i}, i=1, \ldots, N_{\mathrm{t}}$, coupling $N_{\ell}$ rotation-vibration energy levels with energies $E_{j}, j$ $=1, \ldots, N_{\ell}$. The obvious linear relation between transitions and energy levels is

$$
\sigma_{i}=E_{\mathrm{up}(i)}-E_{\mathrm{lo}(i)} .
$$

As emphasized above, it is a strict requirement that the dataset contains a unique assignment to label both the lower (lo) and upper (up) states involved in the transitions. Without this information the inversion process from transitions to energies cannot be executed. A useful choice for the labeling is provided by the usual quantum numbers, the standard normal coordinate labeling (e.g., $n_{1} n_{2} n_{3}$ in the case of water, where $n_{1}, n_{2}$, and $n_{3}$ stand for the symmetric stretching, bending, and antisymmetric stretching quantum numbers, 
in order) for the vibrational states and the standard rotational notation (e.g., $J_{K a K c}$ in the case of asymmetric tops, like water) for the rotational states. For a triatomic molecule, like water, this consists of six labels altogether. The inversion process can of course be used to determine the experimental energy levels for different electronic states. In this case one needs one more unique label to identify the different electronic states.

Two further comments on the choice of labels. First, a relatively good model must be available prior to using MARVEL in order to give proper and unique labels for the experimental transitions. Approximate Hamiltonians as well as variational computations based on highly accurate potential energy surfaces (PES), obtained perhaps $a b$ initio $[8,10,11]$, are able to provide these labels for most small molecules of interest. Second, any labeling scheme other than that presented, even just assigning a simple numerical value to the energy levels if they are all known from a computation, would be sufficient if the uniqueness of the labeling can be ensured.

In order to help critical evaluation and tracking of the energy levels even after a prolonged time, the dataset must also contain information about the sources of the measured transitions. This can be given as metadata, one particular choice, used within MARVEL, involves the published source of the transition and the number of the transition in the given publication. If the source and its labeling do not change over time, which can hardly be ensured with electronic sources but is fulfilled with results published in traditional journals, it is possible to track the transitions in the future, as well.

\subsection{Spectroscopic networks (SN)}

The principal dataset may contain more than one so-called spectroscopic network (SN). Based on simple quantum mechanical selection rules, it is easy to determine which rotationalvibrational transitions and energy levels belong to a particular SN. Of course, the SN used in a particular application of MARVEL is determined by the availability of measured and assigned transitions and will grow in size with time as new measurements become available.

In the case of the $\mathrm{H}_{2}{ }^{17} \mathrm{O}$ isotopologue of water, and indeed for all other symmetrically substituted isotopologues of water, the observed transitions can be divided unequivocally into two main SNs, para and ortho [12]. Those energy levels belong to the para SN for which the number $\left(K_{a}+K_{c}+n_{3}\right)$ is even. By construction, the lowest level within each SN has a value of zero, so that the energy zero is defined unequivocally and with an associated uncertainty of 
zero. MARVEL results in the correct energy levels in an absolute sense for a higher-energy $\mathrm{SN}$ only if the value of the lowest-energy level within the higher-energy SN, zero by definition of the SN, is changed to the correct transition energy. Since, due to the appropriate selection rules, this transition cannot be measured directly (otherwise the two SNs would not be distinct, see, though, below), the difference, which might be called a 'magic number', must be estimated based on empirical and/or theoretical considerations. However, due to the availability of highly accurate approximate Hamiltonians for smaller molecules, this usually presents no hindrance in the determination of highly accurate absolute energy levels for distinct SNs.

We must note at this stage that for water high- $J$ energy levels have a lot of (near) degeneracies between states with ortho and para symmetry [12]. Certainly, for $\mathrm{H}_{2}{ }^{16} \mathrm{O}$ many accidental degeneracies have been noted [15]. In principle one could use these observations to fix the ortho/para magic number. However, it seems more justified, and it is certainly easier, to use this possibility as a control test on whether the original choice of the magic number was correct and not to use it in a direct fashion to merge distinct SNs.

Due to the nature of the spectroscopic measurements, the grand list of experimental transitions may contain so-called floating SNs (FSN) which involve transitions which have no linkage with the origins of the theoretically distinct $\mathrm{SNs}$ (in the case of $\mathrm{H}_{2}{ }^{17} \mathrm{O}$, neither with the origin of the para nor with the origin of the ortho SN). The correct absolute values of these FSN energy levels cannot be computed with MARVEL, just their relative values within the FSN, because the separation of the lowest energy levels of the FSN from the ZPE of the SN is not known. These FSNs can be divided into two parts, to disjoint sets and to orphans. If a given energy level has only one partner and this energy level and its partner belong to one and only one transition then these two energy levels are called orphans. Energy levels which are members of none of the principal SNs and are not orphans either are placed in one of perhaps many FSNs. The purpose of an underlying tree-building algorithm within MARVEL is to put each energy level into one of the transition families (SNs, FSNs, and orphans). After this classification one is almost ready to execute the inversion process to obtain the experimental energy levels. However, a preprocessing of the transition families, especially that of the $\mathrm{SN}(\mathrm{s})$, is needed in order to ensure that only those transitions are used in MARVEL which can help in obtaining accurate energy levels with uncertainties as dependable as possible. 


\subsection{Preprocessing: cleansing of the database}

A general problem concerning data collection is the need to weed out incorrect entries. Errors can be introduced in the assignment procedure and during transcription of the measured data into the database. Finding worst offenders breaking the self-consistency of the database is another problem often requiring specialized solutions. A particular problem arises when a database is created with a collection of measured transitions from different sources, since the same transitions may have been measured by different groups, and the data could have been obtained under significantly different experimental conditions. There are several possibilities for the introduction of systematic inconsistencies.

Preprocessing of multiple measurements of the same transition helps in checking for consistency between the different data sources. Automatic identification of multiple measurement entries is relatively easy when the energy levels involved are well characterized which is typical of those with small quantum numbers. For transitions between higher-lying energy levels this problem might be much more acute and needs particular attention and a lot of experience on the part of the database builder. One possible way to deal with multiple entries is to merge them into a single value with an uncertainty reflecting the confidence of the database builder in the accuracy of the chosen value. On the other hand, MARVEL allows the use of all multiple measurements during the refinement and inversion processes and thus these entries need no special treatment. At present, this seems to be the preferred way to treat such data.

As a next step toward cleansing of the database, it is worth making the distinction between actual misassignments, where completely the wrong quantum numbers have been given to a transition, and mislabelings, where the rigorous quantum numbers for the levels taking part in the transition would be correct but the approximate ones are wrong or different from ones given to another transition involving the same level(s).

One way to identify misassigned transitions is through the generation of transitions from the inverted energy levels. Given the computed uncertainties of the energy levels, and that of the transition in question, one can decide whether a transition identified as misassigned should be deleted from the dataset or it is sufficient if its uncertainty is systematically increased.

Mislabeling can be quite common, especially in water, for which a lot of transitions have been measured by many groups using slightly different approximate quantum labels. To correct for mislabeling requires special attention as it cannot be straightforwardly automated. 
This is especially true if one trawls backwards in the literature. For doubtful cases traditional techniques based on combination differences should certainly be helpful.

The larger the dataset the more often one will run into these difficulties. Correcting these problems for the large number of measured transitions, in cases running into the hundreds of thousands, must be done in a more or less automatic fashion. Over time MARVEL runs help in reducing the chances of misassignment and mislabeling and keeping correct track of the proposed corrections. A particularly promising and constantly improving possibility to identify misassignments and mislabelings is through comparison with levels and transitions coming from independent highly accurate theoretical calculations, e.g., BT2 [13] or CVRQD $[8,10]$. In practice we advocate using results from both spectroscopically determined (e.g., empirically adjusted) and ab initio potential energy surfaces.

A straightforward way to identify unrealistic uncertainties is through the generation of transitions from the inverted energy levels. Division of the difference between the measured and computed transitions, $\Delta_{i}$ [Eq. (8), vide infra], by the experimental uncertainty, $\delta_{i}$, provides a number for each transition which is a reasonable measure of the correctness of the transition. If this number, $\Delta_{i} / \delta_{i}$, is much greater than 1 , this indicates that the given transition is an offender. MARVEL performs a preliminary test to filter the worst offenders out. Those transitions are deleted from the database for which $\Delta_{i} / \delta_{i}$ are greater than a preset "cut-off" parameter. The suggested values for this parameter are 10-100; the stricter value of 10 has been used in the final run for $\mathrm{H}_{2}{ }^{17} \mathrm{O}$.

\subsection{Inversion from transitions to energy levels}

Using a SN, the following overdetermined system of linear equations can be written,

$$
\mathbf{a X}=\mathbf{Y}
$$

where vector $\mathbf{Y}$ (with dimension $N_{\mathrm{t}}$ ) contains the $N_{\mathrm{t}}$ measured transitions of the $\mathrm{SN}$ considered and vector $\mathbf{X}$ (with dimension $N_{\ell}-1$ ) contains the $E_{j}$ energy levels as results. The elements of the input matrix a, with dimension $N_{\mathrm{t}} \times\left(N_{\ell}-1\right)$, are chosen to be $-1,+1$, or 0 according to the following scheme,

$$
a_{i j}=\left\{\begin{array}{l}
-1, \text { if } E_{j} \text { is the lower level of the } i \text { th transition } \\
+1, \text { if } E_{j} \text { is the upper level of the } i \text { th transition } \\
0, \text { otherwise }
\end{array}\right.
$$


The weighted least-squares solution of Eq. (2) can be obtained by solving

$$
\mathbf{A X}=\mathbf{B}
$$

where $\mathbf{A}=\mathbf{a}^{T} \mathbf{g a}, \mathbf{B}=\mathbf{a}^{T} \mathbf{g Y}$, and $g_{i}=1 / \delta_{i}^{2}$, where $\delta_{i}$ is the uncertainty of the $i$ th transition. The dimension of the extremely sparse matrix $\mathbf{A}$ is $\left(N_{\ell}-1\right) \times\left(N_{\ell}-1\right)$ and Eq. (4) is a simple system of linear equations which can be solved much more easily than the overdetermined system of linear equations, Eq. (2).

\subsection{Uncertainties and local sensitivities of energy levels}

Determination of the experimental energy levels is useful in itself, but it is highly desirable that the inversion process yields meaningful estimates for the uncertainties of the energy levels. There is no unique way to compute the uncertainties of the levels. Therefore, in what follows we give several different methods how the uncertainties of the levels can be and have been determined. Each method is useful under different conditions. The first two methods are analytic, and thus preferred, while two others are based on the so-called bootstrap technique [14].

It is possible, through Eq. (4), to determine the uncertainty for each MARVEL energy level. Let $\varepsilon_{j}$ denote the uncertainty associated with $E_{j}$. The simplest way to compute uncertainties for the levels is via the expression [1]

$$
\sigma_{j}^{(1)}=\sqrt{A_{j j}^{-1}}
$$

Each $\varepsilon_{j}^{(1)}$ can be written as $\varepsilon_{j}^{(1)}=t \sigma_{j}^{(1)}$, where $\sigma_{j}^{(1)}$ is the standard deviation. For $t=2$, $\varepsilon_{j}^{(1)}$ represents a $\sim 95 \%$ confidence limit. Each MARVEL energy level can be written as

$$
E_{j} \pm \varepsilon_{j}^{(1)} .
$$

If one could remeasure all the transitions many times, the observation weightings were correct, the observations were coming from a normal distribution, and the correlation between the levels is ignored, the standard deviation of the resulting distributions of energy levels should be given by Eq. (5). Unfortunately, it is not clear that in a practical situation all these criteria hold. Thus, the correctness of all the uncertainties of the large number of measured transitions cannot be ensured and Eq. (5) usually provides optimistic uncertainties for the MARVEL levels. As a simple test shows, even if just one of the transitions is completely wrong or has a way too small uncertainty, this uncertainty estimate for the levels will not be able to call attention to these problems as the uncertainty of the levels remains too small. 
Flaud and co-workers [5] advocated the use of a slightly more complex indicator for the confidence interval,

$$
\varepsilon_{j}^{(2)}=t \sqrt{\left(\mathbf{A}^{-1}\right)_{j j} \frac{\sum_{i} g_{i} \Delta_{i}^{2}}{N_{\mathrm{t}}-N_{\ell}}},
$$

where $\Delta_{i}$ is the difference between the original measured and the computed transition,

$$
\Delta_{i}=\sigma_{i}-\left(E_{\mathrm{up}(i)}-E_{\mathrm{lo}(i)}\right) .
$$

This seems to be the preferred gauge for the uncertainty of the measured energy levels if the dataset may contain transitions of less controlled value or uncertainty, since when $\Delta_{i}$ is much larger than the uncertainty of the $i$ th measured transition it indicates (a) either a misassignment or (b) that a line position is in error. The quantity $\sum_{i} g_{i} \Delta_{i}^{2} /\left(N_{\mathrm{t}}-N_{\ell}\right)$ in Eq. (7) is approximately 1 if $g_{i}=1 / \delta_{i}^{2}$ and thus, under favorable conditions $\varepsilon_{j}^{(1)} \approx \varepsilon_{j}^{(2)}$. As seen in Section 2.6, the condition $\varepsilon_{j}^{(1)} \approx \varepsilon_{j}^{(2)}$ can be achieved after suitable adjustment of the uncertainties of the transitions.

The bootstrap method is a CPU intensive resampling technique introduced by Efron [14] for determining, among other things, standard errors and confidence intervals in statistically complicated situations. Often there are analytical expressions, like Eqs. (5) and (7), to obtain uncertainty estimates, but in case of complex data there might be no dependable analytical formulae available. In these cases the bootstrap technique can be used for determining statistical measures, including uncertainties. There are two types of common bootstrap procedures, (a) resampling cases (RC) and (b) resampling residuals (RS). We employed both methods to estimate the uncertainties of the energy levels. In the case of RC the resampling involves the experimental uncertainties of the transitions, while in the RS case the computed $\Delta_{i}$ are resampled instead. In our algorithm the bootstrap resampling was performed 400 times because in smaller test calculations this choice resulted in near normal distributions for the uncertainties. In runs based on a large number of lines the bootstrap resampling uncertainties were deemed somewhat unrealistic, while running even 400 samplings is expensive. An important result of the test computations is that after appropriate adjustment of the uncertainties of the lines, the condition $\varepsilon_{j}^{(1)} \approx \varepsilon_{j}^{(2)}$ can be fulfilled. Under such circumstances the preferred uncertainty estimate of the MARVEL energy levels is provided either by $\varepsilon_{j}^{(1)}$ or $\varepsilon_{j}^{(2)}$ 
Often times it is useful to know those transitions that influence the uncertainty of a given energy level. This information can be obtained via the computation of the sensitivity matrix, $\mathbf{S}$. The elements of $\mathbf{S}$ can be obtained either numerically and analytically. The numerical calculation is based on the well-known Gauss-type error propagation. The analytical expression, employed in MARVEL, for the sensitivity matrix calculation is

$$
S_{j i}=\frac{\partial E_{j}}{\partial T_{i}}=\sum_{k}^{N_{\ell}-1} \frac{A_{j k}^{-1} a_{i k}}{\delta_{i}^{2}}
$$

In accordance with the usual expectation, the absolute values of the elements of $\mathbf{S}$ are between 0 and 1.

The local sensitivity coefficients provide highly useful information as they tell which transitions have the largest effect on a given rotational-vibrational level. Unfortunately, the larger the transitions database the more expensive is the computation of the elements of the inverse matrix $\mathbf{A}^{-1}$. Consequently, MARVEL can not yield each element of $\mathbf{S}$ when all elements of larger databases are considered.

\subsection{Dealing with unrealistic experimental uncertainties of the transitions}

It is generally appreciated that spectroscopists may provide substantially different uncertainties for their measured transitions. Apart from optimism (or occasional pessimism), the uncertainties within the set of measured transitions assembled from different sources might suffer from sizeable systematic errors. In the case of Fourier transform spectroscopy these include, for example, calibration errors, pressure shifts of an unknown sign, effects arising from the presence of different foreign gases in the sample, and uncertainties related to the automated line center determinations through first and second derivatives. One also should keep in mind that intensities of the lines are not taken into account in the present version of MARVEL. Line intensities can vary many orders of magnitude and in the case of blended or very weak lines the line center determination may suffer from unusually large errors. All these uncertainties can be larger than the intrinsic uncertainty of the transition. Unreliable uncertainties of the experimental transitions will cause problems since the uncertainties attached to the energy levels will greatly depend on the correctness of the uncertainties of the measured transitions, see especially Eq. (7). Therefore, preconditioning of the experimental transitions is a prerequisite of obtaining reliable MARVEL energy levels with reliable and as low as possible uncertainties. 
There are different routes to improve the uncertainties of the transitions database. Since the large number of measured transitions usually precludes a one-by-one analysis of the transition uncertainties, only procedures that can be automated should be considered. From the many strategies possible, including Bayesian analysis, determination of a Nash-Cournot equilibrium, etc., only two have been implemented: the simple worst-offender strategy [1] and robust weighting [9].

The aim of the worst-offender strategy is to modify the uncertainties of the experimental transitions, preferably one by one, so that the experimental and the calculated transitions, within their respected uncertainties, would properly overlap. In each worstoffender cycle one computes the $E_{j}$ energies, the $\varepsilon_{j}$ s according to Eq. 5, and the $\sigma_{i}^{\text {calc }}$ values. If there is no overlap between the measured and the MARVEL levels including the corresponding uncertainties, the experimental uncertainty of the given transition has to be increased by a small amount, the increment chosen was $1 \%$. Occasionally, the difference between $\sigma_{i}$ and $\sigma_{i}^{\text {calc }}$ is much smaller than $\delta_{i}$. Then, the uncertainty of the measured transition can be decreased by a given amount, the increment chosen was $1 \%$. After doing these small corrections for all transitions involved, the energy level calculations are performed again and again with the refined uncertainties until there is no transition in the database for which there is no overlap. At the end, one obtains a self-consistent set of transitions and associated uncertainties. Of course, this iteration can be stopped at will by setting a value for the maximum number of iterations. In case of large datasets this strategy proved somewhat of limited value.

The robust weighting algorithm, advocated by Watson [9], seems to be especially well suited for adjustment of the uncertainties of the measured transitions. It is based on the following simple adjustment formula:

$$
g_{i}=\frac{1}{\delta_{i}^{2}+\alpha \Delta_{i}^{2}}
$$

where $\alpha$ is a positive number $(\alpha \leq 1 / 3)$ chosen for the given problem. A major advantage of the robust weighting algorithm is that it offers a clear choice when the adjustment of the uncertainties of the lines should be stopped. Adjustment of the uncertainties of the lines through this iteratively reweighted least-squares scheme can be stopped when the quantity

$$
\sum_{i} \frac{g_{i} \Delta_{i}^{2}}{N_{\mathrm{t}}-N_{\ell}}
$$


becomes as close to 1 as desired. If this holds, which was achieved in practice after a relatively small number of iterations, then $\varepsilon_{j}^{(1)} \approx \varepsilon_{j}^{(2)}$ is fulfilled.

After cleansing of the database and applying the preferred robust weighting algorithm, MARVEL results in a database containing self-consistent and correctly assigned transitions and the seemingly best possible related uncertainties. Energy levels, and their uncertainties, determined from these transitions are in harmony with the measured transitions and their uncertainties.

\subsection{The computer code MARVEL}

The algorithm presented in the previous subsections was programmed in the $\mathrm{C}++$ language into a code called MARVEL. Using special algorithms to solve Eq. (4), like sparseadaptive Cholesky decomposition, MARVEL can handle even large cases like that presented by the availability of more than 100000 transitions for $\mathrm{H}_{2}{ }^{16} \mathrm{O}$.

MARVEL needs two input files. Ones contains the grand list of transitions, the other contains input parameters for the code, including magic number(s) and cut-off and iteration parameters. The output of MARVEL is a set of transitions with improved uncertainties, a set of MARVEL energy levels with associated uncertainties, and the number of transitions an energy level participates in. All this information is arranged in SNs.

\section{Application of MARVEL to $\mathrm{H}_{2}{ }^{17} \mathrm{O}$}

Due to our long-standing interest in the complete spectroscopy of water $[8,10,15]$, we selected the isotopologue $\mathrm{H}_{2}{ }^{17} \mathrm{O}$ of water as a first test of the utility of the procedure and the code MARVEL.

\subsection{Measured transitions}

The number of all the available measured transitions for $\mathrm{H}_{2}{ }^{17} \mathrm{O}$, in the range of $0-$ $17124.8 \mathrm{~cm}^{-1}$, is 8369 . This range covers 57 vibrational states, from $\left(\begin{array}{lll}0 & 0 & 0\end{array}\right)$ to $\left(\begin{array}{ll}3 & 0\end{array}\right)$ in normal coordinate notation. The transitions data between 0 and $177 \mathrm{~cm}^{-1}$ and between 177 and $600 \mathrm{~cm}^{-1}$ were obtained from Refs. 16- 19. The data in the range 1315 and $1986 \mathrm{~cm}^{-1}$ were obtained from Ref. 20. Data in the ranges $500-7782 \mathrm{~cm}^{-1}$ and $8564-9332 \mathrm{~cm}^{-1}$ were obtained from Refs. 21 and 22, respectively. Data between 4206 and $6600 \mathrm{~cm}^{-1}$ and 6170 and $6747 \mathrm{~cm}^{-1}$ were also obtained from Refs. 23 and 7, respectively. The transitions 
between 9711 and $10883 \mathrm{~cm}^{-1}$ and $11365-14377 \mathrm{~cm}^{-1}$ were obtained from Refs. 24 and 25 , respectively. And finally, the measured transitions between 16570 and $17125 \mathrm{~cm}^{-1}$ were obtained from Ref. 26. The uncertainties of the measured transitions range from $1 \times 10^{-6}$ for the lowest pure rotational levels to $113000 \times 10^{-6}$ for the excitations to levels above 10000 $\mathrm{cm}^{-1}$.

As mentioned before, the measured transitions of the $\mathrm{H}_{2}{ }^{17} \mathrm{O}$ isotopologue of water can be divided into a para and an ortho SN. After cleansing of the database, there are 3205 and 4606 transitions in the para and ortho SNs, respectively. These two SNs determine 1161 and 1575 para and ortho rotational-vibrational levels, respectively.

In the case of $\mathrm{H}_{2}{ }^{17} \mathrm{O}$, the lowest energy level of the ortho $\mathrm{SN}$ is the $101\left(J K_{a} K_{c}\right)$ state. The corresponding "magic number", i.e., the difference between the ZPE of the para SN and the $1_{01}$ state of the ortho SN, $23.773510 \mathrm{~cm}^{-1}$, was obtained from Ref. 21.

\subsection{A small subset of levels determined from transitions of Ref. 19}

In order to show the expected quality of the MARVEL protocol it has been applied first for the $127 \mathrm{H}_{2}{ }^{17} \mathrm{O}$ transitions appearing in Ref. 19. 125 of these transitions have been determined on a spectrometer whose typical accuracy in the far infrared (FIR) region is a mere $10 \mathrm{kHz}$. These rotational transitions on the $\left(\begin{array}{lll}0 & 0 & 0\end{array}\right)$ vibrational ground state have the overall lowest consistent uncertainties among the transitions measured so far for $\mathrm{H}_{2}{ }^{17} \mathrm{O}$. These transitions are listed in Table 1. The measured transitions of Ref. 19 contain three orphans and three FSNs. This is the reason why a few lines have no associated MARVEL levels.

As can be seen from Table 1, when high-quality experimental transitions come from a single source, the MARVEL energy levels can reproduce these transitions almost perfectly. We determined all four uncertainty measures described in section 2.5 for each computed energy levels but in this particular case involving particularly well-defined experimental transitions they yielded basically the same values which has not been the case for other datasets. In this well-defined case of a small dataset, both the worst-offender and the robust weighting algorithms will adjust the uncertainties of the lines to the point that uncertainty measures $\varepsilon_{j}^{(1)}$ and $\varepsilon_{j}^{(2)}$ show the expected agreement to within a few percent. Following a robust fitting adjustment of the line uncertainties, the uncertainty measure $\varepsilon_{j}^{(1)}$ has been used to obtain the uncertainties of the MARVEL transitions reported in Table 1. 
The power of MARVEL is very clear from this small test computation. The energy levels determined by the Hamiltonian model independent MARVEL technique reproduce the measured transitions considerably more accurately, on average almost an order of magnitude better, than the approximate Watson-type A-reduced Hamiltonian, containing 37 parameters, used in Ref. 19. There are only a small number of cases when the difference between the observed and calculated transitions from MARVEL is worse than that given in Ref. 19. In summary, MARVEL clearly confirms that almost all the rotational transitions reported Ref. 19 could be used as measurement standards in the FIR region.

\subsection{MARVEL energy levels with uncertainties of $\mathrm{H}_{2}{ }^{17} \mathrm{O}$}

Some of the transitions available from the literature for $\mathrm{H}_{2}{ }^{17} \mathrm{O}$, detailed in section 3.1 , proved to be faulty, either due to misassignment, mislabeling, or transcription error. These transitions were simply deleted from the SNs. Since the robust-weighting strategy improved the uncertainty estimates of many of the experimental transitions, the transitions we believe are dependable, within the given uncertainties, for $\mathrm{H}_{2}{ }^{17} \mathrm{O}$ are listed in the Supplementary Material. To facilitate the later use of these transitions in MARVEL, they are given after separating them into SNs. The availability of this improved list of transitions for $\mathrm{H}_{2}{ }^{17} \mathrm{O}$ is one of the useful results of the MARVEL process.

Due to their large number, the calculated MARVEL energy levels, with their associated uncertainties, determined for the para and ortho SNs, as well as the FSNs of $\mathrm{H}_{2}{ }^{17} \mathrm{O}$, can be found only in the Supplementary Material. Table 2 lists those vibrational levels of $\mathrm{H}_{2}{ }^{17} \mathrm{O}$ which were determined through MARVEL. Clearly, the number of MARVEL vibrational energy levels is rather limited and the lack of levels is becoming more and more prominent as the energy increases. The uncertainties of the MARVEL vibrational levels are small, for most levels they are better than $10^{-3} \mathrm{~cm}^{-1}$. During the robust weighting procedure producing the entries given in Table 2 and the Supplementary Material $\alpha=0.01$ was used.

Finally, to show the effectiveness of the robust weighting procedure, one can search for any of a large number of small loops within the SNs or even across the SNs. The A = $\left(\begin{array}{llllll}0 & 0 & 0 & 0 & 0 & 0\end{array}\right)$ to $\left(\begin{array}{llllll}0 & 1 & 1 & 1 & 0 & 1\end{array}\right)$ transition exemplifies the virtues of the MARVEL procedure. This transition energy has been measured directly. Alternatively, the higher level can also be

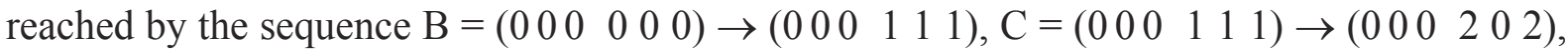

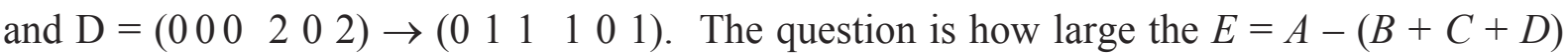
difference is. The measured value of $E$ is a substantial $0.00255 \mathrm{~cm}^{-1}$. After just a single 
MARVEL cycle, i.e., without any adjustment of the uncertainties of the lines, $E$ drops to $-0.00056 \mathrm{~cm}^{-1}$. After the robust reweighting adjustment of the measured uncertainties, i.e., after utilizing the full MARVEL protocol, $E$ becomes an astonishingly small $-0.000065 \mathrm{~cm}^{-1}$, almost two orders of magnitude smaller than the original difference. While this is a nice example to show one of the advantages of the MARVEL procedure, MARVEL's principal virtue is that it allows the joint refinement of all these small loops in one grand weighted least-squares fit.

\section{Summary}

This paper describes the development of a method that obtains energy levels of molecules based on measured transitions. The protocol and the code, both named MARVEL for Measured Active Rotational-Vibrational Energy Levels, allow the determination of a consistent set of energy levels, with associated uncertainties, of small molecules and at the same time results in the improvement of the usually overly optimistic uncertainties of the experimental transitions. Except in the lowest-energy region, the protocol is not particularly sensitive to the fact that experimental measurements come from different sources with different, sometimes conflicting uncertainties though the experimental lines should preferably be adjusted for, for example, calibration differences and pressure shifts. Treatment of multiple measurements for the same transition does not cause problems for MARVEL. The MARVEL protocol allows one to easily and quickly detect misassigned or mislabeled transitions, thus allowing for efficient cleansing of the dataset of experimental lines. This cleansing is important as MARVEL only works best if the transitions are properly assigned prior to running MARVEL, the associated uncertainties are correct, and the dataset contains no outliers. Adjustment of the line uncertainties can be achieved effectively through the preferred robust-weighting strategy. After adjustment of the line uncertainties by the robust weighting protocol, the uncertainties provided by the simple analytic $\varepsilon_{j}^{(1)}$ and $\varepsilon_{j}^{(2)}$ formulas become highly similar and thus either of these two could be used. The bootstrap uncertainties investigated proved to be too expensive to compute and in questionable cases seemingly less dependable than the uncertainties obtained through the use of the $\varepsilon_{j}^{(1)}$ or $\varepsilon_{j}^{(2)}$ formulas.

Particular advantages of determining energy levels through MARVEL include the possibility of a rapid update with new information in a straightforward and statistically meaningful way, easy execution of tests and identification of those transitions whose 
determination would improve most easily the understanding of the energy spectrum of the isotopologue in question, and the availability of full covariance and sensitivity matrices.

As an application of MARVEL, the presently available best set of transitions and energy levels have been determined for the $\mathrm{H}_{2}{ }^{17} \mathrm{O}$ singly-substituted isotopologue of water. A list of 2736 dependable energy levels, based on 8369 transitions, has been obtained. Furthermore, the accuracy of almost all of the $127 \mathrm{H}_{2}{ }^{17} \mathrm{O}$ transitions appearing in Ref. 19 advocated as frequency standards in the far-infrared region has been confirmed.

\section{Acknowledgments}

The research performed in Hungary received support from the Hungarian Scientific Research Fund (OTKA T047185) and from the NKTH. The joint work between Budapest and London received support from the European Union through the MC RTN QUASAAR. Discussions with Drs. Tamás Turányi (ELTE, Budapest, Hungary), Branko Ruscic (ANL, U.S.A), and Linda Brown (JPL, U.S.A.) on the topic of this paper are gratefully acknowledged. A referee of the manuscript is also thanked for useful criticism. This work was performed as part of the activities of the IUPAC Task Group 2004-035-1-100. 
Table 1. Accurate experimentally measured rotational transitions on the ground vibrational state of $\mathrm{H}_{2}{ }^{17} \mathrm{O}$, with assignments and uncertainties (in parentheses), and reproduction of the lines obtained with an $A$-reduced Watson Hamiltonian given as Obs - Calc, all taken from Ref. 19, and associated MARVEL Obs - Calc values, with related uncertainties in parentheses.

\begin{tabular}{|c|c|c|c|c|c|c|c|c|c|}
\hline \multirow{2}{*}{ upper } & \multirow{2}{*}{ lower } & \multirow{2}{*}{ Trans. energy/MHz } & \multicolumn{2}{|c|}{$(\mathrm{Obs}-\mathrm{Calc}) / \mathrm{MHz}$} & \multirow{2}{*}{ upper } & \multirow{2}{*}{ lower } & & (Obs & $/ \mathrm{MHz}$ \\
\hline & & & Ref. 19 & MARVEL & & & & Ref. 19 & MARVEL \\
\hline "616 & 523 & 13535.510 & 0.051 & $0.000(57)$ & 616 & 505 & 3644995.072(39) & 0.063 & $-0.004(57)$ \\
\hline 313 & 220 & 194002.290 & 0.050 & $-0.002(42)$ & 853 & 844 & $3668047.672(60)$ & 0.137 & $0.000(91)$ \\
\hline 423 & 330 & $469809.339(351)$ & -0.589 & $-0.523(50)$ & 762 & 717 & $3683082.986(39)$ & 0.046 & $-0.010(63)$ \\
\hline 110 & 101 & $552021.075(38)$ & 0.010 & $-0.070(30)$ & 423 & 312 & $3787242.695(44)$ & -0.154 & $-0.021(49)$ \\
\hline 532 & 441 & $658504.180(149)$ & -0.360 & $-0.347(65)$ & 615 & 524 & $3803428.532(77)$ & -0.074 & $0.022(57)$ \\
\hline 211 & 202 & $748458.779(42)$ & 0.162 & $0.008(42)$ & 651 & 642 & $3876375.114(85)$ & 0.075 & $0.009(71)$ \\
\hline 422 & 331 & 944853.071(42) & -0.020 & $-0.119(53)$ & 550 & 541 & $3904093.322(156)$ & 0.022 & $0.000(106)$ \\
\hline 524 & 431 & $987879.876(39)$ & -0.151 & $-0.003(53)$ & 652 & 643 & $3908469.423(53)$ & -0.052 & $-0.181(79)$ \\
\hline 202 & 111 & $991519.683(36)$ & 0.004 & $0.060(34)$ & 753 & 744 & $3909669.422(80)$ & -0.509 & $0.000(82)$ \\
\hline 312 & 303 & $1096415.186(36)$ & 0.110 & $-0.006(46)$ & 551 & 542 & $3911126.846(60)$ & 0.199 & $0.000(69)$ \\
\hline 111 & 000 & $1107166.987(36)$ & 0.091 & $0.000(18)$ & 725 & 634 & $4026370.245(48)$ & 0.050 & $0.000(69)$ \\
\hline 321 & 312 & $1148974.962(36)$ & 0.023 & $-0.002(47)$ & 1047 & 1038 & $4036624.943(46)$ & 0.333 & FSN3 \\
\hline 312 & 221 & $1168135.700(36)$ & 0.009 & $-0.010(44)$ & 937 & 928 & $4063443.292(56)$ & -0.115 & $0.000(174)$ \\
\hline 634 & 541 & $1189418.871(38)$ & 0.044 & $0.000(70)$ & 1056 & 1047 & $4082007.509(64)$ & -0.155 & FSN3 \\
\hline 422 & 413 & $1197609.827(36)$ & 0.123 & $-0.048(51)$ & 707 & 616 & $4158000.015(77)$ & -0.173 & $0.018(64)$ \\
\hline 220 & 211 & $1212979.348(36)$ & -0.086 & $0.002(43)$ & 717 & 606 & $4180385.495(48)$ & -0.147 & $-0.016(61)$ \\
\hline 744 & 651 & $1214991.983(51)$ & 0.218 & $0.003(73)$ & 524 & 413 & $4197019.729(42)$ & 0.180 & $0.204(52)$ \\
\hline 827 & 734 & $1282726.792(42)$ & 0.016 & $0.019(75)$ & 827 & 818 & $4231907.193(39)$ & -0.077 & $-0.006(77)$ \\
\hline 743 & 652 & $1325632.503(38)$ & -0.061 & $-0.027(81)$ & 1028 & 1019 & $4351161.455(48)$ & -0.358 & Orp \\
\hline 625 & 532 & $1332129.386(38)$ & 0.117 & $0.038(64)$ & 330 & 303 & $4413848.064(42)$ & 0.066 & $0.019(47)$ \\
\hline 523 & 514 & $1406448.955(38)$ & 0.147 & $0.001(58)$ & 331 & 220 & $4440837.832(80)$ & 0.030 & $0.007(48)$ \\
\hline 633 & 542 & $1583727.403(38)$ & 0.032 & $-0.027(63)$ & 330 & 221 & $4485568.539(44)$ & -0.073 & $-0.024(45)$ \\
\hline 726 & 633 & $1439891.568(149)$ & -0.112 & $-0.017(63)$ & 716 & 625 & $4535154.497(56)$ & 0.352 & $0.084(66)$ \\
\hline 413 & 404 & $1604180.557(38)$ & -0.033 & $-0.037(49)$ & 963 & 954 & $4561561.243(53)$ & 0.473 & $0.000(17)$ \\
\hline 221 & 212 & $1646398.143(39)$ & 0.014 & $-0.003(40)$ & 1038 & 1029 & $4564109.765(39)$ & 0.156 & FSN3 \\
\hline 212 & 101 & $1662464.158(36)$ & 0.064 & $0.052(27)$ & 862 & 853 & $4613500.984(680)$ & -1.626 & $0.000(350)$ \\
\hline 303 & 212 & $1718118.736(38)$ & -0.007 & $0.072(43)$ & 964 & 955 & $4630833.514(816)$ & 2.090 & Orp \\
\hline 633 & 624 & $1739572.000(36)$ & 0.052 & $0.015(63)$ & 625 & 514 & $4578730.854(60)$ & 0.130 & $-0.030(60)$ \\
\hline 734 & 725 & $1783388.025(36)$ & -0.052 & $0.096(72)$ & 761 & 752 & $4633687.667(123)$ & 0.993 & FSN1 \\
\hline 532 & 523 & $1840152.668(36)$ & 0.021 & $0.086(62)$ & 863 & 854 & $4636270.403(71)$ & -1.265 & FSN2 \\
\hline 322 & 313 & $1906062.231(36)$ & -0.149 & $0.000(43)$ & 660 & 651 & $4636336.821(224)$ & -0.554 & $0.000(133)$ \\
\hline 523 & 432 & $1948277.941(36)$ & 0.145 & $0.027(56)$ & 661 & 652 & $4637408.255(53)$ & -0.411 & $0.000(088)$ \\
\hline 431 & 422 & $2011529.712(36)$ & -0.106 & $-0.059(52)$ & 762 & 753 & $4639564.820(71)$ & 1.211 & $0.000(98)$ \\
\hline 835 & 826 & $2013437.033(51)$ & -0.484 & $0.000(77)$ & 808 & 717 & $4713837.243(214)$ & 0.194 & $0.000(124)$ \\
\hline 413 & 322 & $2088016.649(36)$ & 0.080 & $0.032(47)$ & 818 & 707 & $4723224.243(85)$ & 0.144 & $-0.037(74)$ \\
\hline 313 & 202 & $2155440.437(36)$ & 0.146 & $0.028(41)$ & 918 & 909 & $4757672.856(46)$ & -0.110 & $0.000(87)$ \\
\hline 330 & 321 & $2168457.888(38)$ & -0.094 & $-0.001(48)$ & 926 & 919 & $4793077.382(64)$ & -0.178 & $0.000(69)$ \\
\hline 514 & 505 & $2225010.637(39)$ & -0.105 & $0.025(57)$ & 431 & 404 & $4813320.42(69)$ & 0.308 & $0.180(50)$ \\
\hline 423 & 414 & $2252481.128(36)$ & -0.199 & $0.004(53)$ & 936 & 845 & $4850055.006(44)$ & 0.338 & $-0.000(87)$ \\
\hline 836 & 743 & $2262993.376(46)$ & -0.230 & $-0.010(79)$ & 1129 & 11110 & $4972521.517(99)$ & 0.138 & Orp \\
\hline 945 & 936 & $2287034.489(51)$ & 0.153 & $0.000(93)$ & 726 & 615 & $4977140.283(53)$ & -0.081 & $0.019(62)$ \\
\hline 331 & 322 & $2340773.242(38)$ & 0.060 & $-0.060(49)$ & 826 & 735 & $5003094.161(59)$ & 0.033 & $-0.021(70)$ \\
\hline 725 & 716 & $2353116.027(38)$ & -0.074 & $0.079(70)$ & 432 & 321 & $5077587.318(90)$ & -0.199 & $-0.008(52)$ \\
\hline 404 & 313 & $2389898.207(38)$ & -0.152 & $-0.047(46)$ & 817 & 726 & $5188886.526(39)$ & 0.036 & $0.009(66)$ \\
\hline 844 & 835 & $2406767.120(38)$ & 0.177 & $0.000(84)$ & 422 & 313 & $5191688.836(44)$ & 0.183 & $0.113(47)$ \\
\hline 936 & 927 & $2437474.985(59)$ & 0.201 & $0.000(94)$ & 909 & 818 & $5264693.665(42)$ & -0.417 & $0.000(81)$ \\
\hline 432 & 423 & $2439319.592(38)$ & -0.015 & $0.018(54)$ & 919 & 808 & $5268608.573(48)$ & 0.113 & $0.000(65)$ \\
\hline 533 & 524 & $2609740.484(36)$ & -0.030 & $0.000(56)$ & 770 & 761 & $5294347.567(42)$ & 0.039 & FSN1 \\
\hline 734 & 643 & $2612984.953(51)$ & -0.070 & $-0.006(74)$ & 431 & 322 & $5297156.277(56)$ & 0.186 & $0.014(48)$ \\
\hline 743 & 734 & $2621117.184(39)$ & 0.169 & $0.009(76)$ & 871 & 862 & $5306809.792(90)$ & -0.394 & $0.000(88)$ \\
\hline 414 & 303 & $2631176.821(46)$ & 0.223 & $0.038(50)$ & 872 & 863 & $5307776.850(51)$ & -0.011 & FSN2 \\
\hline 524 & 515 & $2675583.371(39)$ & -0.155 & $-0.012(54)$ & 972 & 963 & $5310760.945(156)$ & 0.627 & $0.000(127)$ \\
\hline 221 & 110 & $2756841.024(39)$ & -0.133 & $-0.083(42)$ & & & & & \\
\hline 642 & 633 & $2842780.905(42)$ & -0.190 & $0.002(65)$ & & & & & \\
\hline 634 & 625 & $2861900.086(36)$ & 0.034 & $-0.025(64)$ & & & & & \\
\hline 615 & 606 & $2886328.247(36)$ & 0.029 & $0.004(59)$ & & & & & \\
\hline 220 & 111 & $2952957.663(38)$ & -0.067 & $-0.077(34)$ & & & & & \\
\hline 514 & 423 & $2981148.445(36)$ & -0.150 & $-0.089(55)$ & & & & & \\
\hline 624 & 533 & $2991364.726(36)$ & -0.102 & $0.015(61)$ & & & & & \\
\hline 505 & 414 & $3008619.064(39)$ & -0.116 & $0.017(55)$ & & & & & \\
\hline 826 & 817 & $3009824.350(71)$ & 0.185 & $0.032(71)$ & & & & & \\
\hline 440 & 431 & $3089686.890(38)$ & 0.076 & $0.000(55)$ & & & & & \\
\hline 515 & 404 & $3125616.717(38)$ & 0.105 & $-0.019(52)$ & & & & & \\
\hline 441 & 432 & $3129925.963(42)$ & 0.060 & $-0.006(60)$ & & & & & \\
\hline 542 & 533 & $3147209.261(36)$ & -0.144 & $-0.015(61)$ & & & & & \\
\hline 625 & 616 & $3158746.404(36)$ & -0.053 & $-0.016(59)$ & & & & & \\
\hline 735 & 726 & $3195616.643(38)$ & 0.116 & $-0.010(65)$ & & & & & \\
\hline 643 & 634 & $3196773.182(36)$ & -0.067 & $-0.032(70)$ & & & & & \\
\hline 744 & 735 & $3298639.736(56)$ & 0.044 & $-0.004(69)$ & & & & & \\
\hline 322 & 211 & $3313043.876(46)$ & -0.178 & $0.007(44)$ & & & & & \\
\hline 845 & 836 & $3468801.961(38)$ & 0.055 & $0.000(40)$ & & & & & \\
\hline 954 & 945 & $3451481.974(428)$ & 1.915 & $0.000(233)$ & & & & & \\
\hline 716 & 707 & $3535900.842(39)$ & 0.427 & $0.012(70)$ & & & & & \\
\hline 606 & 515 & $3592683.645(39)$ & -0.269 & $-0.005(56)$ & & & & & \\
\hline 836 & 827 & $3601383.801(51)$ & -0.045 & $0.013(78)$ & & & & & \\
\hline
\end{tabular}




\section{Footnotes to Table 1.}

${ }^{\mathrm{a}} \mathrm{FSN}=$ floating spectroscopic network; Orp $=$ orphan (see text and the Supplementary Material). Entries given in bold face correspond to cases where the three times the stated experimental uncertainty of the level is smaller than the MARVEL estimate of the transition uncertainty. The uncertainties of the MARVEL transitions were obtained from energy level uncertainties determined through Eq. (5). 
Table 2. MARVEL vibrational energies $\left(\mathrm{cm}^{-1}\right)$, their uncertainties, $\varepsilon_{j}^{(1)}=2 \sigma_{j}^{(1)}$ [Eq. (5)], and the associated number of rovibrational levels of $\mathrm{H}_{2}{ }^{17} \mathrm{O}$, based on the database given in the Supplementary Material

\begin{tabular}{|c|c|c|c|c|c|}
\hline$n_{1} n_{2} n_{3}$ & MARVEL & $\begin{array}{l}\text { No. of } \\
\text { Levels }\end{array}$ & $n_{1} n_{2} n_{3}$ & MARVEL & $\begin{array}{l}\text { No. of } \\
\text { Levels }\end{array}$ \\
\hline 000 & 0.000000 & 296 & 260 & & 1 \\
\hline 010 & $1591.325652(4)$ & 191 & 142 & & 14 \\
\hline 020 & $3144.980451(4)$ & 96 & 043 & & 4 \\
\hline 100 & $3653.142273(10)$ & 116 & 420 & & 55 \\
\hline 001 & $3748.318070(10)$ & 153 & 321 & $16797.167510(988)$ & 60 \\
\hline 030 & & 22 & 401 & $16875.620510(988)$ & 55 \\
\hline 110 & $5227.705613(32)$ & 78 & 500 & & 38 \\
\hline 011 & $5320.250929(20)$ & 147 & 161 & & 1 \\
\hline 040 & & 19 & 081 & & 1 \\
\hline 120 & $6764.725613(942)$ & 59 & 222 & & 1 \\
\hline 021 & $6857.272709(32)$ & 89 & 123 & & 2 \\
\hline 200 & $7193.246623(20)$ & 83 & 302 & & 2 \\
\hline 101 & $7238.713600(20)$ & 102 & & Total: & 2736 \\
\hline 002 & $7431.076113(122)$ & 28 & & & \\
\hline 050 & & 1 & & & \\
\hline 130 & & 3 & & & \\
\hline 031 & & 10 & & & \\
\hline 210 & & 34 & & & \\
\hline 111 & $8792.544310(988)$ & 108 & & & \\
\hline 060 & & 1 & & & \\
\hline 012 & $8982.869213(942)$ & 55 & & & \\
\hline 041 & & 13 & & & \\
\hline 220 & & 12 & & & \\
\hline 121 & $10311.202510(988)$ & 75 & & & \\
\hline 022 & & 1 & & & \\
\hline 300 & & 65 & & & \\
\hline 201 & $10598.475610(988)$ & 102 & & & \\
\hline 102 & $10853.505313(942)$ & 53 & & & \\
\hline 003 & & 46 & & & \\
\hline 131 & $11792.827010(6416)$ & 31 & & & \\
\hline 310 & & 28 & & & \\
\hline 211 & $12132.992610(988)$ & 87 & & & \\
\hline 112 & & 25 & & & \\
\hline 013 & $12541.225510(988)$ & 39 & & & \\
\hline 141 & & 1 & & & \\
\hline 042 & & 1 & & & \\
\hline 320 & & 3 & & & \\
\hline 221 & $13631.499810(1086)$ & 53 & & & \\
\hline 400 & & 29 & & & \\
\hline 071 & $13808.273310(988)$ & 2 & & & \\
\hline 301 & $13812.158110(988)$ & 75 & & & \\
\hline 202 & & 14 & & & \\
\hline 103 & $14296.279510(394)$ & 37 & & & \\
\hline 340 & & 13 & & & \\
\hline 241 & & 6 & & & \\
\hline
\end{tabular}




\section{References}

[1] B. Ruscic, R. E. Pinzon, M. L. Morton, G. Von Laszevski, S. J. Bittner, S. G. Nijsure, K. A. Amin, M. Minkoff, A. F. Wagner, J. Phys. Chem. A 108 (2004) 9979-9997.

[2] B. Ruscic, J. E. Boggs, A. Burcat, A. G. Császár, J. Demaison, R. Janoschek, J. M. L. Martin, M. Morton, M. J. Rossi, J. F. Stanton, P. G. Szalay, P. R. Westmoreland, F. Zabel, T. Bérces, J. Phys. Chem. Ref. Data 34 (2005) 573-656.

[3] R. Feeley, P. Seiler, A. Packard, M. Frenklach, J. Phys. Chem. A 108 (2004) 9573-9583.

[4] M. Frenklach, A. Packard, P. Seiler, R. Feeley, Int. J. Chem. Kinet. 36 (2004) 57-66.

[5] J.-M. Flaud, C. Camy-Peyret, J. P. Maillard, Mol. Phys. 32 (1976) 499-521.

[6] S. A. Tashkun, V. I. Perevalov, J.-L. Teffo, A. D. Bykov, and N. N. Lavrentieva, J. Quant. Spectr. Rad. Transfer 82 (2003) 165 - 196.

[7] P. Macko, D. Romanini, S. N. Mikhailenko, O. V. Naumenko, S. Kassi, A. Jenouvrier, Vl. G. Tyuterev, and A. Campargue, J. Mol. Spectry. 227 (2004) 90 - 108.

[8] O. L. Polyansky, A. G. Császár, S. V. Shirin, N. F. Zobov, P. Barletta, J. Tennyson, D. W. Schwenke, and P. J. Knowles, Science 299 (2003) 539 - 542.

[9] J. K. G. Watson, J. Mol. Spectrosc. 219 (2003) 326 - 328.

[10] P. Barletta, S. V. Shirin, N. F. Zobov, O. L. Polyansky, J. Tennyson, E. F. Valeev, A. G. Császár, J. Chem. Phys. 125 (2006) 204307.

[11] A. G. Császár, W. D. Allen, Y. Yamaguchi, H. F. Schaefer III, in Computational Molecular Spectroscopy, eds. P. Jensen and P. R. Bunker, Wiley, New York, 2000.

[12] A. Miani, J. Tennyson, J. Chem. Phys. 120 (2004) 2732 - 2739.

[13] R. J. Barber, J. Tennyson, G. J. Harris, and R. N. Tolchenov, Mon. Nat. Roy. Astron. Soc. 368 (2006) 1087.

[14] B. Efron and R. Tibshirani, An Introduction to the Bootstrap, Chapman and Hall, London, 1993.

[15] J. Tennyson, N. F. Zobov, R. Williamson, O. L. Polyansky, and P. Bernath, J. Phys. Chem. Ref. Data 30 (2001) 735.

[16] J. Steenbeckeliers, CRAS Paris B273 (1971) 471.

[17] F.C. De Lucia, J. Mol. Spectrosc. 56 (1975) 138 - 145.

[18] J. Kauppinen and E. Kyro, J. Mol. Spectrosc. 84 (1980) 405 - 423.

[19] F. Matsushima, H. Nagase, T. Nakauchi, H. Odashima, and K. Takagi, J. Mol. Spectrosc. 193 (1999) $217-223$. 
[20] G. Guelachvili, J. Opt. Soc. Am. 73 (1983) 137 - 150.

[21] http://mark4sun.jpl.nasa.gov/.

[22] A.-W. Liu, S.-M. Hu, C. Camy-Peyret, J.-Y. Mandin, O. Naumenko, and B. Voronin, J. Mol. Spectry. 237 (2006) $53-62$.

[23] A. Jenouvrier, L. Daumont, L. Regalia-Jarlot, V. G. Tyuterev, M. Carleer, A. C. Vandaele, S. Mikhailenko, S. Fally, J. Quant. Spectrosc. Rad. Transfer 105 (2007) 326 - 355.

[24] C. Camy-Peyret, J.-M. Flaud, J.-Y. Mandin, A. Bykov, O. Naumenko, L. Sinitsa, and B. Voronin, J. Quant. Spectrosc. Rad. Transfer 61 (1999) 795 - 812.

[25] M. Tanaka, O. Naumenko, J. W. Brault, and J. Tennyson, J. Mol. Spectrosc. 234 (2005) $1-9$.

[26] O. Naumenko, M. Sneep, M. Tanaka, S.V. Shirin, W. Ubachs, J. Tennyson, J. Mol. Spectrosc. 237 (2006) 63-69. 